Note

\title{
Synthesis of Neu5Ac-Gal-functionalized gold glyconanoparticles
}

\author{
Lei Zhang ${ }^{\mathrm{a}, \mathrm{b}}$, Guohua Wei ${ }^{\mathrm{a}}$, Yuguo $\mathrm{Du}^{\mathrm{a}, \mathrm{b}, *}$ \\ a State Key Laboratory of Environmental Chemistry and Ecotoxicology, Research Center for Eco-Environmental Sciences, Chinese Academy of Sciences, Beijing 100085, China \\ ${ }^{\mathrm{b}}$ College of Chemistry and Chemical Engineering, Graduate University of Chinese Academy of Sciences, Beijing 100049, China
}

\section{A R T I C L E I N F O}

\section{Article history:}

Received 22 April 2009

Received in revised form 24 June 2009

Accepted 25 June 2009

Available online 1 July 2009

\section{Keywords:}

Sialic acid

Gold glyconanoparticles

Carbohydrate antigen

Click chemistry

\begin{abstract}
A B S T R A C T
Neu5Ac-Gal-containing neoglycoside 1 was convergently synthesized through $\mathrm{Cu}(\mathrm{I})$-catalyzed 1,3-dipolar cycloaddition of methyl (6-azidohexyl 5-acetamido-4,7,8,9-tetra-O-acetyl-3,5-dideoxy-2-O- $\alpha$-D-glycero-D-galacto-2-nonulopyranosyl)uronate and 11-thioacetylundecyl 2,4,6-tri-O-benzoyl-3-O-propargyl- $\beta$ D-galactopyranoside. The stable and water-soluble gold glyconanoparticle $\mathbf{2}(d=3.4 \mathrm{~nm})$ has been successfully prepared from neoglycoside $\mathbf{1}$ and characterized by NMR, IR, and TEM techniques.
\end{abstract}

(c) 2009 Elsevier Ltd. All rights reserved.
The well-known cluster glycoside effect $^{1}$ has spawned the construction of myriad multivalent glycopolymers for the study of carbohydrate-protein interactions. Integrated nanoparticle-biomolecule multifunctional systems constitute useful tools to mimic the behavior of biomolecules in cells, thus helping to explore the mechanisms of biological process with a variety of potential applications. ${ }^{2,3}$ Gold nanoclusters functionalized with carbohydrates provide a well-defined chemical composition to intervene in the cell-cell adhesion and recognition processes where carbohydrates are involved. ${ }^{4-8}$ However, assembly of glyconanoparticles from authentic oligosaccharides are rather difficult due to the insufficient quantities and methodology limitations, while the syntheses of these oligosaccharides are complex and often feature low overall yields. ${ }^{9}$ The mission may be considerably simplified if target structures are represented by pseudo-oligosaccharides or oligosaccharide mimics in which certain glycosidic bonds are substituted with non-glycosidic motifs. ${ }^{10-14}$ Among these efforts, 1,2,3-triazole links have emerged as a popular bridging unit in carbohydrate chemistry because of their facile and efficient method of synthesis, which is referred to as 'click chemistry'. ${ }^{15,16}$ This method is based on $\mathrm{Cu}(\mathrm{I})$-catalyzed Huisgen's 1,3-dipolar cycloaddition ${ }^{17}$ of azides and terminal alkynes, and it has been successfully applied for the synthesis of various glycoconjugates including multivalent glycosides, ${ }^{18,19}$ cyclodextrin analogues, ${ }^{20}$ glycopeptide mimetics, ${ }^{21}$ and glycosidase inhibitors. ${ }^{22}$ Moreover, the triazole core is more than a passive linker because it can participate in hydrogen-bonding and dipole interactions, which can favor the binding to biomolecu-

\footnotetext{
* Corresponding author. Tel.: +86 10 62849126; fax: +86 1062923563 .

E-mail address: duyuguo@rcees.ac.cn (Y. Du).
}

lar targets and improve solubility. ${ }^{23}$ In a collaborative project, we were asked to provide neoglycoside $\mathbf{1}$ and its corresponding gold glyconanoparticle $\mathbf{2}$ (Fig. 1) as the references for bioactivity studies. We here report the synthesis of this Neu5Ac-Gal-coated gold nanoparticle.

The synthesis was first envisaged from the $p$-methoxyphenyl glycoside $3^{24}$ (Scheme 1). Deacetylation of $\mathbf{3}$ with $\mathrm{NaOMe}$ in $\mathrm{MeOH}$ $(\rightarrow \mathbf{4})$, followed by regioselective propargylation of 3-OH via a dibutyltin intermediate, gave the corresponding 3-O-propargyl galactopyranoside $\mathbf{5}$ in $80 \%$ yield. Benzoylation of $\mathbf{5}$ with $\mathrm{BzCl}$ in pyridine and subsequent conversion of anomeric $p$-methoxyphenyl (MP) group to trichloroacetimidate were carried out smoothly in the presence of ceric ammonium nitrate (CAN), generating glycosyl donor $\mathbf{7}$ in a yield of $82.8 \%$ from $\mathbf{5} .^{25}$ Glycosylation of $\mathbf{7}$ with 11 -thioacetylundecanol using TMSOTf as promoter afforded 8 in $95 \%$ yield. ${ }^{26}$ Convergently, glycosylation of $\mathbf{9}^{27}$ with 6-azido-1-hexanol in the presence of NIS and TMSOTf at $-40{ }^{\circ} \mathrm{C}$ gave 10 in $70 \%$ yield. The presence of an $\alpha$-linked sialic acid moiety in $\mathbf{1 0}$ was supported by its ${ }^{1} \mathrm{H}$ NMR spectrum, which was comparable with the NMR signals of an $\alpha$-linked sialic acid moiety in earlier reports. ${ }^{28-30}$ In the ${ }^{1} \mathrm{H}$ NMR spectrum of compound 10, a doublet of doublets at $\delta 2.57$ (dd, $1 \mathrm{H}, J 12.8,4.9 \mathrm{~Hz}, \mathrm{H}-3 \mathrm{e}$ ) indicated the $\alpha$-stereochemistry of the C-2 of sialic acid moiety.

'Click chemistry' of azide $\mathbf{1 0}$ and alkyne $\mathbf{8}$ was induced by $\mathrm{CuSO}_{4}$ and sodium ascorbate in $1: 1 \mathrm{H}_{2} \mathrm{O}-\mathrm{THF}$ at $50-60{ }^{\circ} \mathrm{C},{ }^{31}$ generating the desired 1,2,3-triazole $\mathbf{1 1}$ in $92 \%$ yield (Scheme 2). Saponification of $\mathbf{1 1}$ with a catalytic amount of $\mathrm{NaOMe}$ in $\mathrm{MeOH}$, followed by in situ $\mathrm{O}_{2}$ oxidation, methyl ester deletion in aq $\mathrm{NaOH}$, neutralization with Amberlite IR-120 $\left(\mathrm{H}^{+}\right)$, and purification on a Sephadex LH-20 column, afforded neoglycoside 1 in $80 \%$ yield. Treatment of 1 

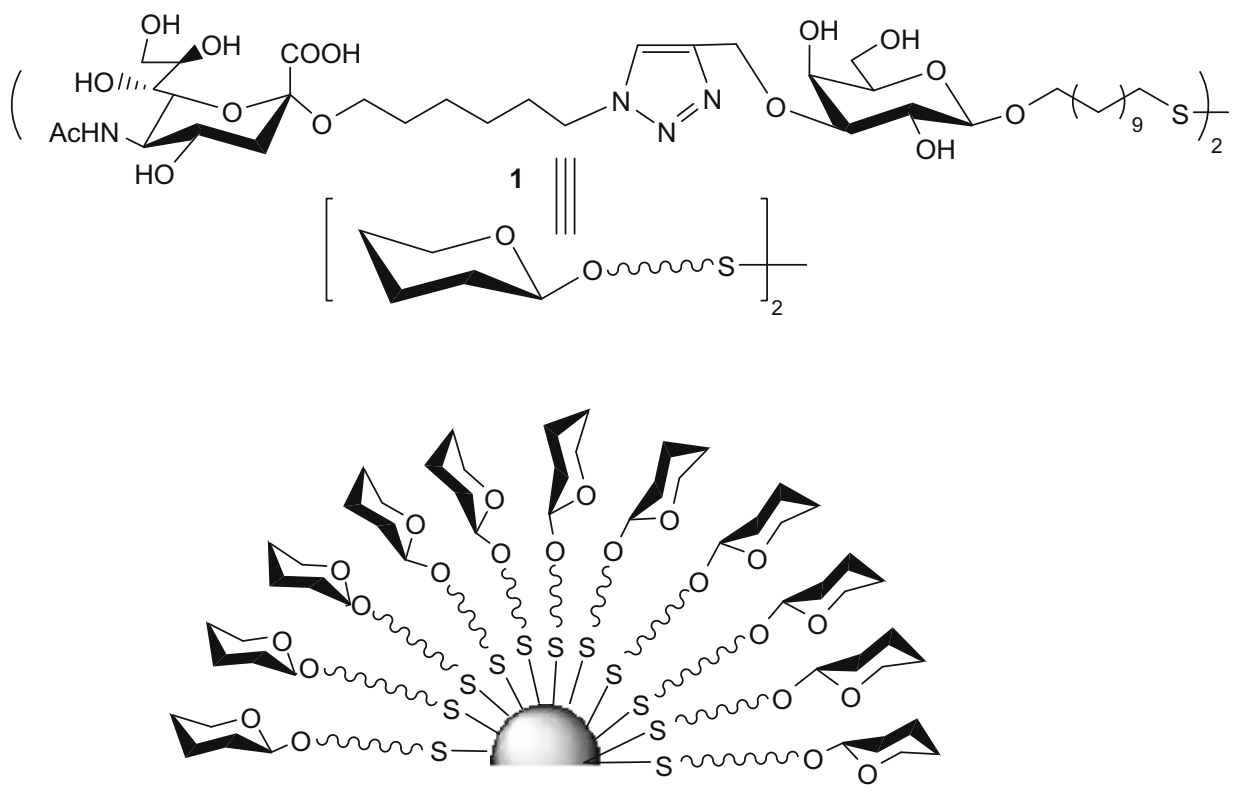

2

Figure 1. Neoglycoside $\mathbf{1}$ and gold glyconanoparticle $\mathbf{2}$.

with $\mathrm{NaBH}_{4}$ and $\mathrm{HAuC}_{4}$, according to a reported procedure, ${ }^{32}$ furnished glyconanoparticle 2 , which was characterized by ${ }^{1} \mathrm{H}$ NMR, and FTIR spectroscopy, and transmission electron microscopy (TEM, Fig. 2). The mean diameter of the generated particles is $3.4 \mathrm{~nm}$, corresponding to an average number of 976 gold atmos. $^{33,34}$ The bioactivity of compounds $\mathbf{1}$ and $\mathbf{2}$ is currently under investigation by our collaborators, and the results will be disclosed in due course.

\section{Experimental}

\subsection{General methods}

Optical rotations were determined at $25^{\circ} \mathrm{C}$ with a Perkin-Elmer model 241-Mc automatic polarimeter; $[\alpha]_{D}$-values are in units of
$10^{-1} \mathrm{deg} \mathrm{cm}^{2} \mathrm{~g}^{-1}$. ${ }^{1} \mathrm{H}$ NMR and ${ }^{13} \mathrm{C}$ NMR spectra were recorded with a Bruker ARX 400 spectrometer for the solutions in $\mathrm{CDCl}_{3}$, DMSO- $d_{6}, \mathrm{CD}_{3} \mathrm{OD}$, or $\mathrm{D}_{2} \mathrm{O}$. The chemical shifts are given in ppm downfield from internal $\mathrm{Me}_{4} \mathrm{Si}$. Mass spectra were measured using a MALDI-TOF mass spectrometer with $\alpha$-cyano-4-hydroxycinnamic acid (CCA) as matrix. FTIR spectra were recorded using a Perkin-Elmer 1720X spectrophotometer by the standard $\mathrm{KBr}$ technique. TLC was performed on silica gel $\mathrm{HF}_{254}$ with detection by charring with $30 \%(\mathrm{v} / \mathrm{v}) \mathrm{H}_{2} \mathrm{SO}_{4}$ in $\mathrm{MeOH}$ or in some cases by a UV lamp. Column chromatography was conducted by elution of a column of silica gel (100-200 mesh) with EtOAc-petroleum ether $\left(60-90^{\circ} \mathrm{C}\right)$ as the eluent. The solutions were concentrated at $<60{ }^{\circ} \mathrm{C}$ under reduced pressure. For TEM examinations, a single drop of the aq solution (ca. $0.1 \mathrm{mg} / \mathrm{mL}$ ) of the gold glyconanoparticles was placed onto a copper grid coated with a carbon film. The grid was left to dry in air for several hours at room temperature.

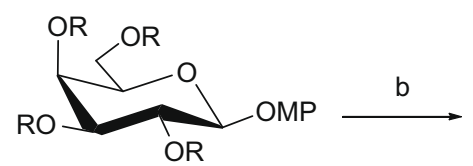

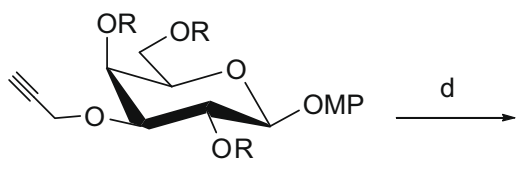

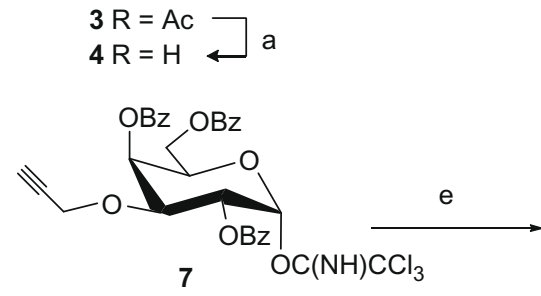

$$
\begin{aligned}
& 5 \mathrm{R}=\mathrm{H} \\
& 6 \mathrm{R}=\mathrm{Bz} \longleftarrow \mathrm{C}
\end{aligned}
$$

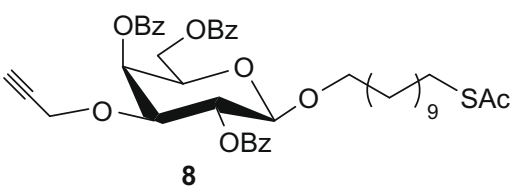

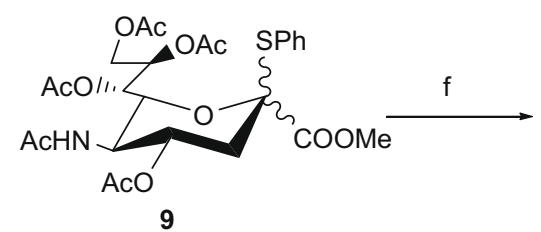

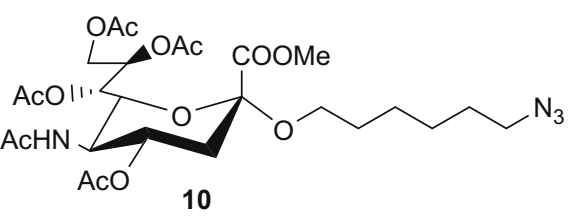

Scheme 1. Reagents and conditions: (a) $\mathrm{MeONa}, \mathrm{MeOH}$, rt; (b) (i) $\mathrm{MeOH}, \mathrm{Bu}_{2} \mathrm{SnO}$, reflux, (ii) propargyl bromide, $\mathrm{Bu}_{4} \mathrm{NI}$, toluene, $60{ }^{\circ} \mathrm{C}, 80 \%$; (c) Pyr, $\mathrm{BzCl}, \mathrm{DMAP}, 92 \%$; (d) (i) CAN, 4:1 $\mathrm{CH}_{3} \mathrm{CN}-\mathrm{H}_{2} \mathrm{O}$; (ii) $\mathrm{Cl}_{3} \mathrm{CCN}$, DBU, $\mathrm{CH}_{2} \mathrm{Cl}_{2}, 90 \%$ from 6; (e) 11-thioacetylundecanol, TMSOTf, $\mathrm{CH}_{2} \mathrm{Cl}_{2}, 0$ ' $\mathrm{C}$, 95\%; (f) 6-azido-1-hexanol, NIS, TMSOTf, 1:1 CH $\mathrm{Cl}_{2}$ - $\mathrm{CH}_{3} \mathrm{CN}_{\text {, }}$ $-40{ }^{\circ} \mathrm{C}, 70 \%$. 


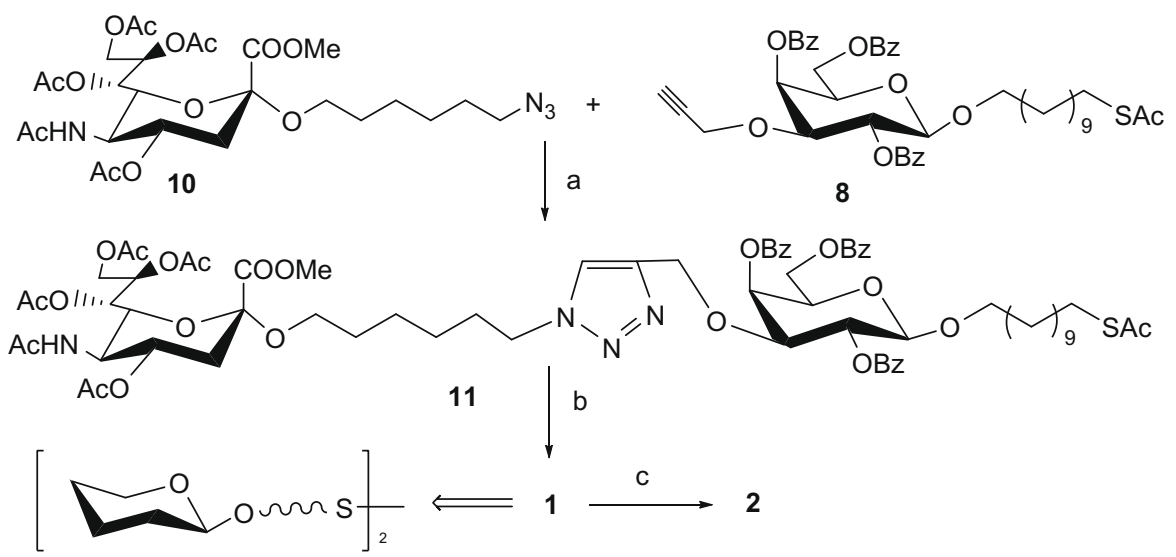

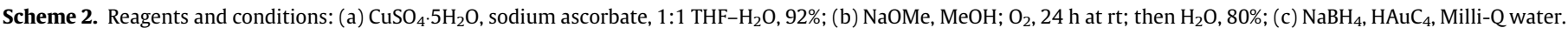

TEM analysis was carried out in a Hitachi H-7500 microscope working at $200 \mathrm{kV}$.

\section{2. -Methoxyphenyl 3-O-propargyl- $\beta$-D-galactopyranoside (5)}

To a solution of 3 (10.5 g, $23.1 \mathrm{mmol})$ in anhyd $\mathrm{MeOH}(100 \mathrm{~mL})$ was added $1 \mathrm{~N} \mathrm{NaOMe}$ in $\mathrm{MeOH}$ until $\mathrm{pH}$ 9.5. The mixture was stirred at $\mathrm{rt}$ for $5 \mathrm{~h}$, then neutralized with Amberlite IR-120 $\left(\mathrm{H}^{+}\right)$and filtered. The filtrate was concentrated to dryness, and the resulting compound 4 was used without further purification. After refluxing of $4(5.521 \mathrm{~g}, 19.28 \mathrm{mmol})$ and dibutyltin oxide $(5.299 \mathrm{~g}$, $21.26 \mathrm{mmol})$ in anhyd $\mathrm{MeOH}(85 \mathrm{~mL})$ under a nitrogen protection for $4 \mathrm{~h}$, the reaction mixture was concentrated under vacuum. The dried residue was suspended in toluene $(136 \mathrm{~mL})$ under nitrogen atmosphere, and propargyl bromide $(4.6 \mathrm{~mL}, 23.19 \mathrm{mmol})$ and tetrabutylammonium iodide $(7.11 \mathrm{~g}, 19.28 \mathrm{mmol})$ were added with vigorous stirring at $60{ }^{\circ} \mathrm{C}$ for $18 \mathrm{~h}$. At the end of this time, TLC indicated that all starting materials were consumed, and the solvent was then evaporated. The residue was purified by silica gel column chromatography (8:1 EtOAc-MeOH) to afford $\mathbf{5}$ $(5.01 \mathrm{~g}, 80 \%)$ as an amorphous white solid: $[\alpha]_{\mathrm{D}}^{25}-8\left(\mathrm{c} 1, \mathrm{CHCl}_{3}\right)$; ${ }^{1} \mathrm{H}$ NMR (400 MHz, CD $\mathrm{CD}_{3}$ ): $\delta 2.86(\mathrm{t}, 1 \mathrm{H}, J 2.4 \mathrm{~Hz}, \equiv \mathrm{CH}), 3.57$ (dd, $1 \mathrm{H}, J$ 9.7, $3.2 \mathrm{~Hz}, \mathrm{H}-3$ ), 3.62 (br d, $1 \mathrm{H}, J 5.9 \mathrm{~Hz}, \mathrm{H}-6 \mathrm{a}), 3.73-$ $3.76\left(\mathrm{~m}, 5 \mathrm{H}, \mathrm{H}-5, \mathrm{H}-6 \mathrm{~b}\right.$, and $\left.\mathrm{OCH}_{3}\right), 3.84(\mathrm{dd}, 1 \mathrm{H}, J 9.7,7.8 \mathrm{~Hz}, \mathrm{H}-$ 2), 4.11 (br d, $1 \mathrm{H}, J 3.1 \mathrm{~Hz}, \mathrm{H}-4), 4.38-4.40\left(\mathrm{~m}, 2 \mathrm{H}, \mathrm{OCH}_{2}\right), 4.75$ (d, $1 \mathrm{H}, J 7.8 \mathrm{~Hz}, \mathrm{H}-1), 6.82,7.05\left(2 \mathrm{~d}, 2 \times 2 \mathrm{H}, \mathrm{C}_{6} \mathrm{H}_{4}\right)$. Anal. Calcd for

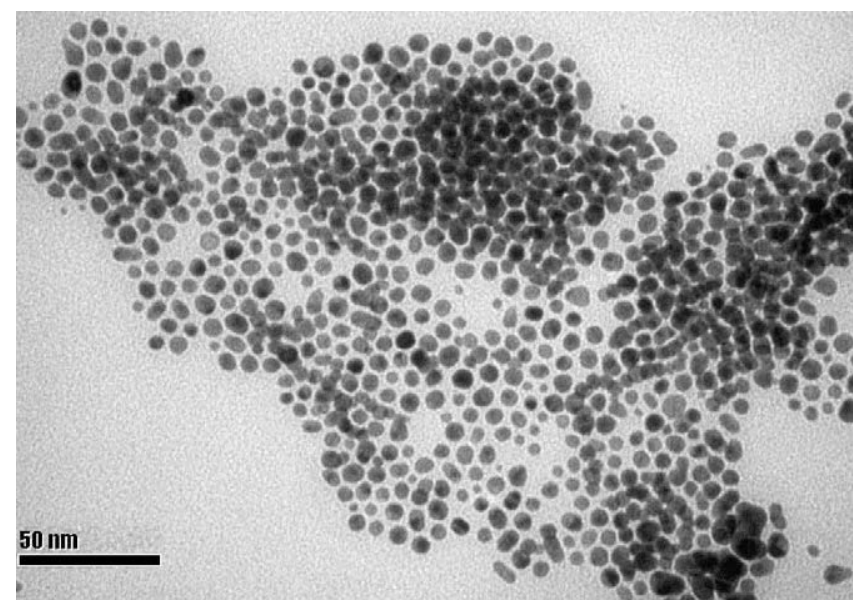

Figure 2. TEM of $\mathbf{2}$ shows the uniform size and dispersion of the particles.
$\mathrm{C}_{16} \mathrm{H}_{20} \mathrm{O}_{7}$ : C, 59.25; H, 6.22. Found: C, 59.04; H, 6.28. MALDI-TOFMS: calcd for $\mathrm{C}_{16} \mathrm{H}_{20} \mathrm{O}_{7}$ : $324.12[\mathrm{M}]^{+}$; found: $347.23[\mathrm{M}+\mathrm{Na}]^{+}$.

\section{3. p-Methoxyphenyl 2,4,6-tri-O-benzoyl-3-O-propargyl- $\beta$-D- galactopyranoside (6)}

To a solution of $5(1.81 \mathrm{~g}, 5.58 \mathrm{mmol})$ in pyridine $(10 \mathrm{~mL})$ were added $\mathrm{BzCl}(2.32 \mathrm{~mL}, 19.9 \mathrm{mmol})$ and DMAP $(30 \mathrm{mg})$ at $0{ }^{\circ} \mathrm{C}$. The reaction mixture was stirred at $\mathrm{rt}$ for $4 \mathrm{~h}$, then quenched by adding $\mathrm{MeOH}(1 \mathrm{~mL})$, and concentrated under diminished pressure. The residue was redissolved in $\mathrm{CH}_{2} \mathrm{Cl}_{2}$ and was washed successively with $1 \mathrm{~N} \mathrm{HCl}(\mathrm{aq})$, aq $\mathrm{NaHCO}_{3}$, and brine. The organic phase was dried over $\mathrm{Na}_{2} \mathrm{SO}_{4}$, and concentrated. Purification of the residue by column chromatography on silica gel (1:3 EtOAc-petroleum ether) afforded $6(3.26 \mathrm{~g}, 92 \%)$ as an amorphous solid: $[\alpha]_{\mathrm{D}}^{25}+72$ (c 0.6, $\mathrm{CHCl}_{3}$ ); ${ }^{1} \mathrm{H} \mathrm{NMR}\left(400 \mathrm{MHz}, \mathrm{CDCl}_{3}\right): \delta 2.34(\mathrm{t}, 1 \mathrm{H}, J 2.3 \mathrm{~Hz}$, $\equiv \mathrm{CH}), 3.71$ (s, 3H, OMe), 4.20-4.27 (m, $4 \mathrm{H}, \mathrm{H}-3, \mathrm{H}-5$, and $\left.\mathrm{OCH}_{2}\right)$, 4.54 (dd, $1 \mathrm{H}, J 11.5,5.2 \mathrm{~Hz}, \mathrm{H}-6 \mathrm{a}), 4.61$ (dd, $1 \mathrm{H}, J 11.5,7.8 \mathrm{~Hz}, \mathrm{H}-$ 6b), 5.15 (d, $1 \mathrm{H}, J 8.0 \mathrm{~Hz}, \mathrm{H}-1$ ), 5.74 (dd, $1 \mathrm{H}, J$ 9.9, $8.0 \mathrm{~Hz}, \mathrm{H}-2$ ), 5.87 (br d, $1 \mathrm{H}, J 2.8 \mathrm{~Hz}, \mathrm{H}-4), 6.65,6.95\left(2 \mathrm{~d}, 2 \times 2 \mathrm{H}, \mathrm{C}_{6} \mathrm{H}_{4}\right), 7.44-$ $8.18(\mathrm{~m}, 15 \mathrm{H}, 3 \mathrm{Ph})$. Anal. Calcd for $\mathrm{C}_{37} \mathrm{H}_{32} \mathrm{O}_{10}: \mathrm{C}, 69.80 ; \mathrm{H}, 5.07$. Found: $\mathrm{C}, 69.62 ; \mathrm{H}, 5.14$. MALDI-TOFMS: calcd for $\mathrm{C}_{37} \mathrm{H}_{32} \mathrm{O}_{10}$ : $636.20[\mathrm{M}]^{+}$; found: $659.27[\mathrm{M}+\mathrm{Na}]^{+}$.

\subsection{2,4,6-Tri-0-benzoyl-3-0-propargyl- $\beta$-D-galactopyranosyl trichloroacetimidate $(7)$}

To a solution of 6 (637 mg, $1 \mathrm{mmol}$ ) in $\mathrm{CH}_{3} \mathrm{CN}(16 \mathrm{~mL})$ and $\mathrm{H}_{2} \mathrm{O}$ $(4 \mathrm{~mL})$ was added ammonium cerium nitrate $(1.64 \mathrm{~g}, 3.0 \mathrm{mmol})$ at rt. After stirring for $1.5 \mathrm{~h}$, the reaction mixture was diluted with EtOAc and $\mathrm{H}_{2} \mathrm{O}$. The organic layer was washed with brine, dried over $\mathrm{Na}_{2} \mathrm{SO}_{4}$, and concentrated. Further purification was carried out by silica gel column chromatography (1:2 EtOAc-petroleum ether) to give a yellowish amorphous solid. To a solution of the above solid (516 mg) in $\mathrm{CH}_{2} \mathrm{Cl}_{2}(3 \mathrm{~mL})$ were added trichloroacetonitrile $(0.3 \mathrm{~mL}, 3 \mathrm{mmol})$ and DBU $(30 \mu \mathrm{L})$ at rt. After stirring for $3 \mathrm{~h}$, the reaction mixture was concentrated, and the residue was purified by silica gel column chromatography (1:4 EtOAc-petroleum ether) to give 7 (594 mg, 90\%) as an amorphous white solid: $[\alpha]_{\mathrm{D}}^{25}+11\left(\right.$ c $\left.0.75, \mathrm{CHCl}_{3}\right) ;{ }^{1} \mathrm{H}$ NMR $\left(400 \mathrm{MHz}, \mathrm{CDCl}_{3}\right): \delta 2.45(\mathrm{t}$, $1 \mathrm{H}, J 2.4 \mathrm{~Hz}, \equiv \mathrm{CH}), 4.27$ (dd, $\left.1 \mathrm{H}, J 16.4,2.3 \mathrm{~Hz}, \mathrm{OCH}_{2}\right), 4.48(\mathrm{dd}, 1$ $\mathrm{H}, J 16.4,2.3 \mathrm{~Hz}, \mathrm{OCH}_{2}$ ), 4.52 (dd, $1 \mathrm{H}, J 11.4,5.5 \mathrm{~Hz}, \mathrm{H}-6 \mathrm{a}$ ), 4.55 (dd, $1 \mathrm{H}, J 11.4,7.0 \mathrm{~Hz}, \mathrm{H}-6 \mathrm{~b}$ ), 4.66 (dd, $1 \mathrm{H}, J 10.3,3.3 \mathrm{~Hz}, \mathrm{H}-3$ ), 4.70 (dd, $1 \mathrm{H}, J 5.5,7.0 \mathrm{~Hz}, \mathrm{H}-5), 5.67$ (dd, $1 \mathrm{H}, J 10.3,3.7 \mathrm{~Hz}, \mathrm{H}-2$ ), $6.02(\mathrm{~d}, 1 \mathrm{H}, J 2.6 \mathrm{~Hz}, \mathrm{H}-4), 6.84(\mathrm{~d}, 1 \mathrm{H}, J 3.7 \mathrm{~Hz}, \mathrm{H}-1), 7.41-8.14$ $(\mathrm{m}, 15 \mathrm{H}, 3 \mathrm{Ph}), 8.57(\mathrm{~s}, 1 \mathrm{H}, \mathrm{NH})$. MALDI-TOFMS: calcd for $\mathrm{C}_{32} \mathrm{H}_{26} \mathrm{Cl}_{3} \mathrm{NO}_{9}$ : $673.07[\mathrm{M}]^{+}$; found: $695.16[\mathrm{M}+\mathrm{Na}]^{+}$. 
1.5. 11-Thioacetylundecyl 2,4,6-tri-O-benzoyl-3-O-propargyl- $\beta$ D-galactopyranoside (8)

To a mixture of 7 (290 mg, $0.438 \mathrm{mmol}), 11$-thioacetylundecanol $(90.14 \mathrm{mg}, 0.366 \mathrm{mmol})$, and $4 \AA$ molecular sieves $(100 \mathrm{mg})$ in anhyd $\mathrm{CH}_{2} \mathrm{Cl}_{2}(5 \mathrm{~mL})$ was added TMSOTf $(7.94 \mu \mathrm{L}, 0.044 \mathrm{mmol})$ at $0{ }^{\circ} \mathrm{C}$ under an $\mathrm{N}_{2}$ atmosphere. The reaction mixture was stirred under these conditions for $1.5 \mathrm{~h}$, then neutralized with $\mathrm{Et}_{3} \mathrm{~N}$ and filtered. The filtrate mixture was concentrated to dryness. The residue was purified by silica gel column chromatography (1:3 EtOAc-petroleum ether) to give $\mathbf{8}(594 \mathrm{mg}, 95 \%)$ as an amorphous solid: $[\alpha]_{\mathrm{D}}^{25}+127\left(\right.$ c $\left.1, \mathrm{CHCl}_{3}\right) ;{ }^{1} \mathrm{H}$ NMR $\left(400 \mathrm{MHz}, \mathrm{CDCl}_{3}\right): \delta 1.08-$ $1.19\left(\mathrm{~m}, 18 \mathrm{H}, 9 \mathrm{CH}_{2}\right), 2.32(\mathrm{~s}, 3 \mathrm{H}, \mathrm{OAc}), 2.33(\mathrm{t}, 1 \mathrm{H}, J 2.4 \mathrm{~Hz}$, $\equiv \mathrm{CH}), 2.85\left(\mathrm{t}, 2 \mathrm{H}, \mathrm{J} 7.2 \mathrm{~Hz}, \mathrm{CH}_{2} \mathrm{~S}\right), 3.49-3.53\left(\mathrm{~m}, 1 \mathrm{H}, \mathrm{OCH}_{2}\right), 3.92-$ $3.96\left(\mathrm{~m}, 1 \mathrm{H}, \mathrm{OCH}_{2}\right), 4.15-4.20(\mathrm{~m}, 3 \mathrm{H}, \mathrm{H}-3, \mathrm{H}-5$, and one proton of $\left.\mathrm{OCH}_{2} \mathrm{C} \equiv \mathrm{CH}\right), 4.29(\mathrm{dd}, 1 \mathrm{H}, J 16.6,2.4 \mathrm{~Hz}$, another proton of $\mathrm{OCH}_{2} \mathrm{C} \equiv \mathrm{CH}$ ), 4.41 (dd, $\left.1 \mathrm{H}, J 11.3,6.3 \mathrm{~Hz}, \mathrm{H}-6 \mathrm{a}\right), 4.63$ (dd, $1 \mathrm{H}, J$ 11.3, $6.7 \mathrm{~Hz}, \mathrm{H}-6 \mathrm{~b}$ ), 4.70 (d, J $8.0 \mathrm{~Hz}, \mathrm{H}-1$ ), 5.48 (dd, 1H, J 10.0, $8.0 \mathrm{~Hz}, \mathrm{H}-2$ ), 5.84 (d, J $3.1 \mathrm{~Hz}, \mathrm{H}-4), 8.00-8.15$ (m, 15H, 3Ph). Anal. Calcd for $\mathrm{C}_{43} \mathrm{H}_{50} \mathrm{O}_{10} \mathrm{~S}$ : C, 68.05; $\mathrm{H}, 6.64$. Found: $\mathrm{C}, 67.81 ; \mathrm{H}, 6.59$. MALDI-TOFMS: calcd for $\mathrm{C}_{43} \mathrm{H}_{50} \mathrm{O}_{10} \mathrm{~S}$ : $758.31[\mathrm{M}]^{+}$; found: 781.27 $[\mathrm{M}+\mathrm{Na}]^{+}$.

\subsection{Methyl (6-azidohexyl 5-acetamido-4,7,8,9-tetra-0-acetyl-3, 5-dideoxy-2-0- $\alpha$-D-glycero-D-galacto-2-nonulopyranosid)uro- nate (10)}

To a stirred mixture of $\mathbf{9}$ (250 $\mathrm{mg}, 0.4284 \mathrm{mmol}), 6$-azido-1hexanol (55.7 mg, $0.43 \mathrm{mmol}$ ), and $4 \AA$ molecular sieves (30 mg) in dry $1: 1 \mathrm{CH}_{2} \mathrm{Cl}_{2}-\mathrm{CH}_{3} \mathrm{CN}(5 \mathrm{~mL})$ were added NIS (131 mg, $0.64 \mathrm{mmol})$ and TMSOTf ( $7 \mu \mathrm{L}, 0.043 \mathrm{mmol})$ at $-40{ }^{\circ} \mathrm{C}$. The reaction mixture was stirred under these conditions for $6 \mathrm{~h}$ until all starting materials were consumed. The mixture was then neutralized with $\mathrm{Et}_{3} \mathrm{~N}$, and filtered, and the filtrate was concentrated to dryness. The residue was purified by silica gel column chromatography $(2: 1$ EtOAc-petroleum ether) to give $\mathbf{1 0}(186 \mathrm{mg}, 70 \%)$ as an amorphous solid: $[\alpha]_{\mathrm{D}}^{25}-12\left(\right.$ c $\left.1, \mathrm{CHCl}_{3}\right) ;{ }^{1} \mathrm{H}$ NMR $\left(400 \mathrm{MHz}, \mathrm{CDCl}_{3}\right): \delta 1.35-$ $1.39(\mathrm{~m}, 4 \mathrm{H}), 1.57-1.61(\mathrm{~m}, 4 \mathrm{H}), 1.86,2.00,2.01,2.06,2.12(5 \mathrm{~s}$, $15 \mathrm{H}), 2.57$ (dd, $1 \mathrm{H}, J 12.8,4.9 \mathrm{~Hz}), 3.27-3.29(\mathrm{~m}, 3 \mathrm{H}), 3.46-3.48$ $(\mathrm{m}, 1 \mathrm{H}), 3.79(\mathrm{~s}, 3 \mathrm{H}), 3.91(\mathrm{dd}, 1 \mathrm{H}, J 10.5,2.3 \mathrm{~Hz}), 4.09-4.11(\mathrm{~m}$, $2 \mathrm{H}), 4.80(\mathrm{dd}, 1 \mathrm{H}, J 12.3,2.4 \mathrm{~Hz}), 5.17-5.19(\mathrm{~m}, 1 \mathrm{H}), 5.25-5.27$ $(\mathrm{m}, 1 \mathrm{H}), 5.36-5.38(\mathrm{~m}, 1 \mathrm{H}), 5.41$ (br d, $1 \mathrm{H}, J 10.0) ;{ }^{13} \mathrm{C}$ NMR $\left(100 \mathrm{MHz}, \mathrm{CDCl}_{3}\right): \delta 18.8,18.9,19.1,21.1,23.7,24.4,26.7,27.3$, 35.5, 47.5, 49.4, 50.7, 60.5, 61.9, 66.6, 66.9, 69.8, 70.2, 74.8, 75.1, 75.4, 96.5, 165.6, 166.6, 168.2, 168.6, 168.8, 169.1. Anal. Calcd for $\mathrm{C}_{26} \mathrm{H}_{40} \mathrm{~N}_{4} \mathrm{O}_{13}$ : C, 50.64; H, 6.54. Found: C, 50.51; H, 6.59. MALDITOFMS: calcd for $\mathrm{C}_{26} \mathrm{H}_{40} \mathrm{~N}_{4} \mathrm{O}_{13}: 616.26[\mathrm{M}]^{+}$; found: 639.37 $[\mathrm{M}+\mathrm{Na}]^{+}$.

1.7. 11-Thioacetylundecyl 3-0-[(methyl 5-acetamido-4,7,8,9-tetra-0-acetyl-3,5-dideoxy-2-0- $\alpha$-D-glycero-D-galacto-2-nonulopyranosylhexanyl)triazol-1-methyl]-2,4,6-tri-O-benzoyl- $\beta$-D-galactopyranoside (11)

To a suspension of $\mathbf{1 0}(266 \mathrm{mg}, 0.43 \mathrm{mmol})$ and $\mathbf{8}(272 \mathrm{mg}$ $0.4313 \mathrm{mmol})$ in $1: 1 \mathrm{H}_{2} \mathrm{O}-\mathrm{THF}(40 \mathrm{~mL})$ were added sodium ascorbate (34.18 mg, $0.17 \mathrm{mmol}$ ) and $\mathrm{CuSO}_{4} \cdot 5 \mathrm{H}_{2} \mathrm{O}$ (21.4 mg, $0.086 \mathrm{mmol}$ ) under vigorous stirring. The mixture was stirred in a dark room at $50-60{ }^{\circ} \mathrm{C}$ until complete consumption of the reactants was indicated by TLC analysis. The solvent was evaporated, and the residue was diluted with EtOAc, and washed with $\mathrm{H}_{2} \mathrm{O}$ and brine. The aq layer was extracted with EtOAc, and the combined organic layers were dried over $\mathrm{Na}_{2} \mathrm{SO}_{4}$ and concentrated. Further purification was carried out by silica gel column chromatography (3:1 EtOAc-petroleum ether) to give 11 (545 mg, 92\%) as a white amorphous solid: $[\alpha]_{\mathrm{D}}^{25}+70\left(c \quad 0.6, \mathrm{CHCl}_{3}\right) ;{ }^{1} \mathrm{H}$ NMR $\left(400 \mathrm{MHz}, \mathrm{CDCl}_{3}\right): \delta 1.05-1.52(\mathrm{~m}, 26 \mathrm{H}), 1.85,1.951 .99,2.01$ $2.29(5 \mathrm{~s}, 15 \mathrm{H}), 2.10(\mathrm{t}, 4 \mathrm{H}, J 5.3 \mathrm{~Hz}), 2.55(\mathrm{dd}, 1 \mathrm{H}, J 12.8,4.8 \mathrm{~Hz})$, $2.83(\mathrm{t}, 2 \mathrm{H}, J 7.3 \mathrm{~Hz}), 3.22-3.24(\mathrm{~m}, 1 \mathrm{H}), 3.45-3.47(\mathrm{~m}, 1 \mathrm{H}), 3.75$ (s, 3H), $3.88(\mathrm{t}, 1 \mathrm{H}, J 3.8 \mathrm{~Hz}), 4.07-4.10(\mathrm{~m}, 7 \mathrm{H}), 4.33$ (dd, 1H, J $12.8,1.8 \mathrm{~Hz}), 4.60-4.63(\mathrm{~m}, 2 \mathrm{H}), 4.81-4.83(\mathrm{~m}, 2 \mathrm{H}), 5.29(\mathrm{dd}, 1 \mathrm{H}$, $J$ 10.7, $2.1 \mathrm{~Hz}$ ), 5.35 (br d, $1 \mathrm{H}, J 5.8 \mathrm{~Hz}), 5.43-5.45(\mathrm{~m}, 2 \mathrm{H}), 5.84$ (br d, $1 \mathrm{H}, J 8.6 \mathrm{~Hz}), 7.41-8.13(\mathrm{~m}, 16 \mathrm{H}) ;{ }^{13} \mathrm{C} \mathrm{NMR}\left(100 \mathrm{MHz}, \mathrm{CDCl}_{3}\right.$ ): $\delta$ 21.3, 21.4, 21.5, 21.6, 23.6, 23.7, 25.6, 25.7, 26.3, 29.4, 29.6, 29.7, 29.9, 30, 31.2, 38.1, 38.6, 49.8, 49.9, 53.1, 53.2, 62.8, 62.9, 63, 63.1, $62.2,65.2,67.9,69,69.4,69.5,69.7,70.972 .3,72.6,77.3,77.7,78$, $99.1,102.1,129,129.1,130.2,130.4,130.6,133.7,133.8,134$, $165.7,166.4,166.6,168.2,170.6,170.7,171.1,171.2,171.3$, 196.6. Anal. Calcd for $\mathrm{C}_{69} \mathrm{H}_{90} \mathrm{~N}_{4} \mathrm{O}_{23} \mathrm{~S}$ : C, 60.25; H, 6.59. Found: $\mathrm{C}$, 60.39; $\mathrm{H}, 6.67$. MALDI-TOFMS: calcd for $\mathrm{C}_{69} \mathrm{H}_{90} \mathrm{~N}_{4} \mathrm{O}_{23} \mathrm{~S}: 1374.57$ $[\mathrm{M}]^{+}$; found: $1397.46[\mathrm{M}+\mathrm{Na}]^{+}$.

\subsection{1,11'-Dithiobis\{undecyl [3-0-[(5-acetamido-3,5-dideoxy- 2-0- $\alpha$-D-glycero-D-galacto-2-nonulopyranosylhexanyl)triazol-1- methyl]-p-D-galactopyranoside]\} (1)}

To a solution of $\mathbf{1 1}(100 \mathrm{mg}, 0.07 \mathrm{mmol})$ in anhyd $\mathrm{MeOH}$ $(20 \mathrm{~mL})$ was added $1 \mathrm{~N}$ NaOMe until pH 9.5. The reaction mixture was stirred at rt for $40 \mathrm{~h}$, then a gentle stream of oxygen was bubbled through the solution, and stirring was continued for approximately $24 \mathrm{~h}$, at the end of which time $\mathrm{H}_{2} \mathrm{O}(3 \mathrm{~mL})$ was added. After stirring for another $12 \mathrm{~h}$, the reaction mixture was cooled to $0{ }^{\circ} \mathrm{C}$, neutralized with amberlite IR-120 $\left(\mathrm{H}^{+}\right)$, and then filtered, and the filtrate was evaporated. The residue was purified with Sephadex LH-20 and the desired fractions were lyophilized to give $\mathbf{1}$ $(48.7 \mathrm{mg}, 80 \%)$ as a white amorphous solid: $[\alpha]_{\mathrm{D}}^{25}+90$ (c 0.5 , $\left.\mathrm{H}_{2} \mathrm{O}\right) ;{ }^{1} \mathrm{H}$ NMR $\left(400 \mathrm{MHz}, 49: 1\right.$ DMSO- $\left.d_{6}-\mathrm{D}_{2} \mathrm{O}\right): \delta$ 1.11-1.46 (m, $26 \mathrm{H}), 1.72-1.74(\mathrm{~m}, 2 \mathrm{H}), 1.84(\mathrm{~d}, 3 \mathrm{H}, J 7.0 \mathrm{~Hz}), 2.59$ (dd, $1 \mathrm{H}, J$ 10.8, $1.8 \mathrm{~Hz}), 2.61(\mathrm{t}, 2 \mathrm{H}, J 7.1 \mathrm{~Hz}), 3.14-3.25(\mathrm{~m}, 3 \mathrm{H}), 3.30-3.38$ $(\mathrm{m}, 3 \mathrm{H}), 3.46-3.74(\mathrm{~m}, 5 \mathrm{H}), 3.86(\mathrm{br} \mathrm{s}, 1 \mathrm{H}), 4.06(\mathrm{~d}, 1 \mathrm{H}, J 7.7 \mathrm{~Hz})$, 4.25-4.27 (m, 2H), $4.53(\mathrm{dd}, 1 \mathrm{H}, J 12.2,2.1 \mathrm{~Hz}), 4.64(\mathrm{~d}, 1 \mathrm{H}, J$

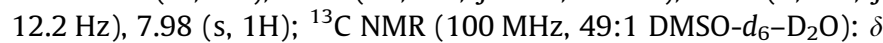
$28.6,29.5,29.9,30,30.2,30.6,39,5,39.7,39.9,40.2,40.3,40.6$, $40.8,50.4,61.2,62.4,63,63.2,63.8,64,65.5,69.6,69.8,71.4$, $72.3,72.5,73.4,75.8,82.3,101.7,104.2,124.7,145.6,172.1$, 173.6; IR (KBr): $\gamma=3352,2927,2855,1717,1626,1437,1377$, 1072, $1035 \mathrm{~cm}^{-1}$. Anal. Calcd for $\mathrm{C}_{74} \mathrm{H}_{130} \mathrm{~N}_{8} \mathrm{O}_{30} \mathrm{~S}_{2}$ : C, 53.03; $\mathrm{H}$, 7.82. Found: $\mathrm{C}, 52.84 ; \mathrm{H}$, 7.87. MALDI-TOFMS: calcd for $\mathrm{C}_{74} \mathrm{H}_{130} \mathrm{~N}_{8} \mathrm{O}_{30} \mathrm{~S}_{2}$ : $1674.83[\mathrm{M}]$; found: $1675.98[\mathrm{M}+\mathrm{H}]^{+}, 837.92$ $[(\mathrm{M}+\mathrm{H}) / 2]^{+}$.

\subsection{Synthesis of GNP (2)}

To a solution of disulfide $\mathbf{1}$ (30 mg, $0.018 \mathrm{mmol}$ ) in Milli-Q $\mathrm{H}_{2} \mathrm{O}$ $(4 \mathrm{~mL})$ was added $\mathrm{HAuCl}_{4} \cdot 4 \mathrm{H}_{2} \mathrm{O}(22.11 \mathrm{mg})$. The solution was stirred at rt for $10 \mathrm{~min}$, kept at $0{ }^{\circ} \mathrm{C}$ for $15 \mathrm{~min}$, then $\mathrm{NaBH}_{4}(3.585 \mathrm{mg}$, $0.0087 \mathrm{mmol}$, in $0.5 \mathrm{~mL} \mathrm{H}_{2} \mathrm{O}$ ) was added in small portions under vigorous stirring conditions. The reaction mixture turned immediately to deep brown. The mixture was kept under these conditions for another $15 \mathrm{~min}$, then warmed up to $\mathrm{rt}$ and stirred for further $3 \mathrm{~h}$. At the end of this time, the solvent was removed by centrifugation, and the black residue was redissolved in $\mathrm{H}_{2} \mathrm{O}(12 \mathrm{~mL})$ and purified by centrifugal filtering ( $40 \mathrm{~min}, 14,000 \mathrm{rpm}$ ). The process was repeated three times. Finally, the $\mathrm{H}_{2} \mathrm{O}$ phase was lyophilized to afford the gold glyconanoparticles $\mathbf{2}(10 \mathrm{mg})$ as a dark brown powder. Average diameter and number of gold atoms: $3.4 \mathrm{~nm}$, 976. ${ }^{1} \mathrm{H}$ NMR $\left(400 \mathrm{MHz}, \mathrm{D}_{2} \mathrm{O}\right): \delta 1.12-1.80(\mathrm{~m}, 26 \mathrm{H}), 1.95-1.97$ $(\mathrm{m}, 2 \mathrm{H}), 2.08-2.11(\mathrm{~m}, 3 \mathrm{H}), 2.42(\mathrm{dd}, 1 \mathrm{H}, J 4.6,1.8 \mathrm{~Hz}), 2.59$ (dd, $1 \mathrm{H}, J 1.8,2.1 \mathrm{~Hz}), 2.94(\mathrm{t}, 2 \mathrm{H}, J 8.3 \mathrm{~Hz}), 3.51(\mathrm{br} \mathrm{d}, 1 \mathrm{H}, J 9.0 \mathrm{~Hz}$ ), 3.59-3.96 (m, 12H), 4.16-4.19 (m, 2H), 4.48-4.50 (m, 1H), 8.10 (s, 1H); IR (KBr): $\gamma=3395,2928,2855,1711,1635,1415,1118$, 1082, 1044. Found analytical data for 2: C, 23.70; H, 3.36. 


\section{Acknowledgment}

This work was supported in partial by NNSF of China (Projects 20872172, 20732001, and 20621703).

\section{Supplementary data}

Supplementary data associated with this article can be found, in the online version, at doi:10.1016/j.carres.2009.06.039.

\section{References}

1. Lundquist, J. J.; Toone, E. J. Chem. Rev. 2002, 102, 555-578.

2. Niemeyer, C. M. Angew. Chem., Int. Ed. 2001, 40, 4128-4158.

3. Katz, E.; Willner, I. Angew. Chem., Int. Ed. 2004, 43, 6042-6108.

4. Penadés, S.; Martin-Lomas, M. Tetrahedron: Asymmetry 2005, 16, 149-158.

5. de la Fuente, J. M.; Barrientos, A. G.; Rojas, T. C.; Rojo, J.; Canada, J.; Fernandez, A.; Penadés, S. Angew. Chem., Int. Ed. 2001, 40, 2258-2261.

6. Carvalho de Souza, A.; Halkes, K. M.; Meeldij, J. D.; Verkleij, A. J.; Vliegenthart, J. F. G.; Kamerling, J. P. ChemBioChem. 2005, 6, 828-831.

7. de la Fuente, J. M.; Eaton, P.; Barrientos, A. G.; Menendez, M.; Penades, S. J. Am Chem. Soc. 2005, 127, 6192-6197.

8. Rojo, J.; Diaz, V.; de la Fuente, J. M.; Segura, I.; Barrientos, A. G.; Riese, H.-H. Bernad, A.; Penadés, S. ChemBioChem. 2004, 5, 291-297.

9. Seeberger, P. H. Chem. Soc. Rev. 2008, 37, 19-28.

10. Du, Y.; Linhardt, R. J.; Vlahov, I. R. Tetrahedron 1998, 54, 9913-9959.

11. Greffe, L.; Jensen, M. T.; Chang-Pi-Hin, F.; Fruchard, S.; O’Donohue, M. J.; Svensson, B.; Driguez, H. Chem. Eur. J. 2002, 8, 5447-5455.

12. Rostovtsev, V. V.; Green, L. G.; Fokin, V. V.; Sharpless, K. B. Angew. Chem., Int. Ed. 2002, 41, 1596-2599.
13. Jiménez Blanco, J. L.; Bootello, P.; Benito, J. M.; Ortiz Mellet, C.; Garcia Fernández, J. M. J. Org. Chem. 2006, 71, 5136-5143.

14. Ramamoorthy, P. S.; Gervay, J. J. Org. Chem. 1997, 62, 7801-7805.

15. Kolb, H. C.; Finn, M. G.; Sharpless, K. B. Angew. Chem., Int. Ed. 2001, 40, 20042021.

16. Meldal, M.; Tornøe, C. W. Chem. Rev. 2008, 108, 2952-3015.

17. Huisgen, R.. In Padwa, A., Ed.; 1,3-Dipolar Cycloaddition Chemistry; Wiley: New York, 1984; Vol. 1, pp 1-176.

18. Chittaboina, S.; Xie, F.; Wang, Q. Tetrahedron Lett. 2005, 46, 2331-2336.

19. Tejler, J.; Tullberg, E.; Frejd, T.; Leffler, H.; Nilsson, U. J. Carbohydr. Res. 2006, 341, 1353-1362.

20. Bodine, K. D.; Gin, D. Y.; Gin, M. S. Org. Lett. 2005, 7, 4479-4482.

21. Fernandez-Megia, E.; Correa, J.; Rodriguez-Meizoso, I.; Riguera, R. Macromolecules 2006, 39, 2113. 2120

22. Rossi, L. L.; Basu, A. Bioorg. Med. Chem. Lett. 2005, 15, 3596-3599.

23. Horne, W. S.; Yadav, M. K.; Stout, C. D.; Ghadiri, M. R. J. Am. Chem. Soc. 2004, 126, 15366-15367.

24. Bazin, H. G.; Du, Y.; Polat, T.; Linhardt, R. J. J. Org. Chem. 1999, 64, 7254-7259.

25. Schmidt, R. R.; Beyerbach, A. Liebigs Ann. Chem. 1992, 983-986.

26. Hiyai, G.; Watanabe, T.; Yamaguchi, K.; Miyagi, T.; Sodeoka, M. J. Am. Chem. Soc. 2007, 129, 15420-15421.

27. Marra, A.; Sinaÿ, P. Carbohydr. Res. 1989, 181, 35-42.

28. Castro-Palomino, J. C.; Ritter, G.; Fortunato, S. R.; Reinhardt, S.; Old, LJ.; Schmidt, R. R. Angew. Chem., Int. Ed. 1997, 26, 1998-2001.

29. Ikeda, K.; Sugiyama, Y.; Tanaka, K.; Sato, M. Bioorg. Med. Chem. Lett. 2002, 12, 2309-2311.

30. Kumar, P.; Misra, A. K. Tetrahedron 2008, 64, 8685-8691.

31. Chen, Q.; Yang, F.; Du, Y. Carbohydr. Res. 2005, 340, 2476-2482.

32. Brust, M.; Walter, M.; Berthell, D.; Schiffrin, D. J.; Whyman, R. J. Chem. Soc., Chem. Commun. 1994, 801-802.

33. Hostetler, M. J.; Wingate, J. E.; Zhong, C.-J.; harris, J. E.; Vachet, R. W.; Clark, M. R.; Londono, J. D.; Green, S. J.; Stokes, J. J.; Wignall, G. D.; Glish, G. L.; Porter, M. D.; Evans, N. D.; Murray, R. W. Langmuir 1998, 14, 17-30.

34. Ojeda, R.; de Paz, J. L.; Barrientos, A. G.; Martin-Lomas, M.; Penadés, S. Carbohydr. Res. 2007, 342, 448-459. 